\title{
Studies on serum soluble endoglin: An indicator for preeclampsia
}

\author{
Archana $\mathrm{A}^{1, *}$, Brindha $\mathrm{G}^{2}$, Ursula Sampson ${ }^{3}$ \\ ${ }^{\mathbf{1}}$ Assistant Professor, ${ }^{2,3}$ Professor and HOD, Dept. of Biochemistry, ${ }^{\mathbf{1}, \mathbf{2}}$ ACS Medical College \& Hospital, Velappanchavadi, \\ Chennai, Tamil Nadu, ${ }^{3}$ Meenakshi Medical College \& Research Institute, Kanchipuram, Tamil Nadu, India
}

*Corresponding Author: Archana A

Email: archanaselvakumar08@gmail.com

Received: $20^{\text {th }}$ June, 2018

Accepted: $11^{\text {th }}$ July, 2018

\begin{abstract}
Introduction: To study the concentration of serum soluble endoglin during second trimester pregnancy and monitor for the development of preeclampsia.

Materials and Methods: Seventy-five healthy singleton pregnant women in second trimester was evaluated in this study. ELISA technique was used for measuring the concentration of Serum soluble endoglin. Patients with increased level of serum endoglin were monitored for signs and symptoms of preeclampsia.

Result: Thirty five pregnant women developed preeclampsia among 75 pregnant women. The mean value for sENG in cases was $8.7 \pm 1.4$ and control $5.9 \pm 0.6$. The normal value of serum sENG varies from 2.54 to $7.06 \mathrm{ng} / \mathrm{ml}$. Hence, it is higher than the normal limit in cases.

Conclusions: The concentration of sENG was studied and women were monitored for development of preeclampsia. The present study showed that women with increased serum soluble endoglin levels developed preeclampsia later. Hence, it is suggested that soluble endoglin will be useful in predicting pre eclampsia.
\end{abstract}

Keywords: Endoglin, Preeclampsia, ELISA, sENG.

\section{Introduction}

Preeclampsia (PE) is a pregnancy specific disorder with hypertension and proteinuria after 20 weeks gestational age. ${ }^{1-3}$ It occurs in approximately 3-5\% of pregnancies. ${ }^{4}$ Globally it is one of the major causes for foetal and maternal morbidity and mortality.

Preeclampsia is defined as the presence of hypertension with systolic blood pressure greater than $140 \mathrm{mmHg}$ or diastolic blood pressure more than 90 $\mathrm{mmHg}$ and proteinurea greater than $0.3 \mathrm{~g} /$ day after the twentieth week of pregnancy in a usually normotensive woman. ${ }^{3}$ Odema is one of the symptoms of preeclampsia. Many theories were found for the cause of preeclampsia during the research carried in $21 \mathrm{st}$ century. Such theories are related to mechanisms involving oxidative stress, immunologic intolerance between the fetoplacental unit and maternal tissue, and angiogenic imbalance. The endoglin protein, which is involved in regulation of placental trophoblast differentiation/invasion of the uterus, represents an antiangiogenic factor potentially involved in preeclampsia development given that placental and blood pressure abnormalities are observed in preeclampsia. ${ }^{5}$

Hypertension in pregnancy is split into four main categories such as gestational hypertension, pre eclampsia, chronic hypertension (essential or secondary) and pre-eclampsia superimposed on chronic hypertension. Chronic hypertension is defined as hypertension diagnosed before the 20th gestational week or de novo hypertension, which fails to settle post-partum. Gestational hypertension is defined as de novo arterial hypertension (SBP $\geq 140 \mathrm{mmHg}$ and / or $\mathrm{DBP} \geq 90 \mathrm{mmHg}$ on 2 occasions $>6$ hours apart) occurring after gestational week 20 , which returns to normal post-partum. A sudden increase in BP or proteinuria, or the appearance of thrombocytopenia or deranged transaminases are said to be suggestive but not diagnostic of superimposed preeclampsia.

Pre-eclampsia may be life threatening for both mother and child, increasing the rate of both maternal and fetal morbidity and mortality. After pre eclamptic pregnancies, the mother may develop premature cardiovascular disease, such as chronic hypertension, ischemic heart disease, and stroke, later in life. The children born to preeclamptic mother are relatively small at birth. In addition, the children have an increased risk of developing stroke, coronary heart disease, and metabolic syndrome in adult life.

Preeclamptic women express pathophysiological changes in angiogenic biomarkers which have been reported recently. ${ }^{6}$ In women with severe and/or early onset preeclampsia the concentration of angiogenic factors like Vascular Endothelial Growth Factor and Placental Growth Factor were found to be low. The anti angiogenic factors like soluble fms like tyrosine kinase1 and soluble Endoglin levels were increased. Endoglin, also called CD105, is one such anti angiogenic factor expressed highly on endothelial cells and on placental syncytiotrophoblasts. Endoglin acts as a co-receptor for the transforming growth factor family. ${ }^{7}$ Soluble endoglin is released into the maternal circulation as a result the placental endoglin is up regulated in preeclampsia. By few weeks' time this peptide precedes the onset of hypertension and proteinuria and correlates with the severity of the disease. In 2006, Venkatesha et al., ${ }^{8}$ reported that soluble form of endoglin was present 
in the sera of pregnant women, elevated in preeclamptic individuals and correlated with disease severity. Indeed, the vascular endothelium is considered as the target organ of the biochemical alterations in the placenta during pre-eclampsia. Pre-eclampsia is associated with increase in anti-angiogenic substances. This phenomenon could possibly be detectable in the maternal circulation already in early pregnancy preceding the development of the symptoms. Therefore, it provides a predictive tool for identifying women at risk.

Recently, several studies have been reported that two antiangiogenic peptides such as sFlt-1 and soluble endoglin concentration in serum have been elevated in women with established preeclampsia due to its secretion from the placenta. There are few studies which suggest that the serum levels of these peptides are significantly higher in women with severe preeclampsia than in women with mild disease. ${ }^{9}$ The pathogenesis behind these studies would be, Soluble endoglin, an antiangiogenic molecule which originates from placenta is secreted in increased level. This may be the reason for the pathogenesis of pre-eclampsia. Transforming growth factor $\beta 1$ is responsible for angiogenesis and keeping the lining of the blood vessels healthy. This TGF $\beta 1$ is antagonized by soluble endoglin. As a result, there is increased blood pressure as the cells lining the blood vessels begin to sicken and die. In addition there is leakage of protein into the tissues and urine from the blood vessel.

\section{Materials and Methods}

On approval from ethical committee, the clinical study was conducted on pregnant mothers in the
Department of Clinical Biochemistry, Meenakshi Medical College, Hospital \& Research Institute, Kanchipuram, Tamilnadu. Blood samples from women between 19-29 years of age were taken in this study. Totally 75 healthy singleton pregnant women between 16 weeks to 20 weeks with no underlying medical illness were considered for this study. Only primigravida mothers were included in the study. Multiparity and multiple pregnancies were excluded. The serum soluble endoglin level was estimated and patients were grouped into control and cases that developed preeclampsia.

After a rest of 15 minutes, the BP of each woman was measured using the auscultatory method with a standardized calibrated mercury column type sphygmomanometer with an appropriate sized cuff encircling at least $80 \%$ of the arm in the seated posture, with feet on the floor and arm supported at heart level. At the time of enrolment in the study, all women underwent full history taking. Ultrasonography was done to confirm the gestational age and to rule out congenital fetal abnormalities. Collected maternal blood samples were allowed to clot then serum was separated by centrifugal speed of $2000 \mathrm{rpm}$ for 10 minutes. Serum was removed, placed in eppendorf tubes and stored at $80^{\circ} \mathrm{C}$ until estimation of serum soluble endoglin by ELISA.

\section{Results}

Out of 75 pregnant women, 40 patients did not develop any symptoms of pre eclampsia whereas 35 patients had symptoms of preeclampsia. Table 1 represents the mean SBP, DBP and serum soluble endoglin levels.

Table 1: Demographic characteristics

\begin{tabular}{|l|c|c|c|}
\hline \multicolumn{1}{|c|}{ Parameters } & Control group & Preeclampsia & p value \\
\hline Age $(\mathrm{yrs})$ & $23.5 \pm 2.5$ & $24.1 \pm 2.2$ & 0.00 \\
\hline SBP $(\mathrm{mm} \mathrm{Hg})$ & $106.6 \pm 4.5$ & $134.5 \pm 4.0$ & $<0.01$ \\
\hline DBP $(\mathrm{mm} \mathrm{Hg})$ & $72.3 \pm 3.3$ & $89.8 \pm 2.9$ & $<0.01$ \\
\hline Serum soluble endoglin $(\mathrm{ng} / \mathrm{ml})$ & $5.9 \pm 0.6$ & $8.7 \pm 1.4$ & $<0.01$ \\
\hline
\end{tabular}

Systolic Blood Pressure in Cases and Controls: The mean SBP was $134.5 \pm 4.0$ in cases and $106.6 \pm 4.5$ in controls and represented in Fig. 1. The mean sENG was $5.9 \pm 0.6$ in controls and $8.7 \pm 1.4$ in pre eclampsia. The $\mathrm{p}$ value was calculated for SBP for cases and controls, which was 0.01 .

Diastolic Blood Pressure in Cases and Controls: In Fig. 2, the mean diastolic blood pressure was $89.8 \pm 2.9$ in cases and $72.3 \pm 3.3$ in controls. The $p$ value for DBP was 0.01 , which is significant.

Serum Soluble Endoglin Levels in Cases and Controls: The mean sENG in cases was $8.7 \pm 1.4$ and control $5.9 \pm 0.6$. The normal value of serum sENG varies from 2.54 to $7.06 \mathrm{ng} / \mathrm{ml}$ as shown in Fig. 3 . Hence, it is higher than the normal limit in cases. 


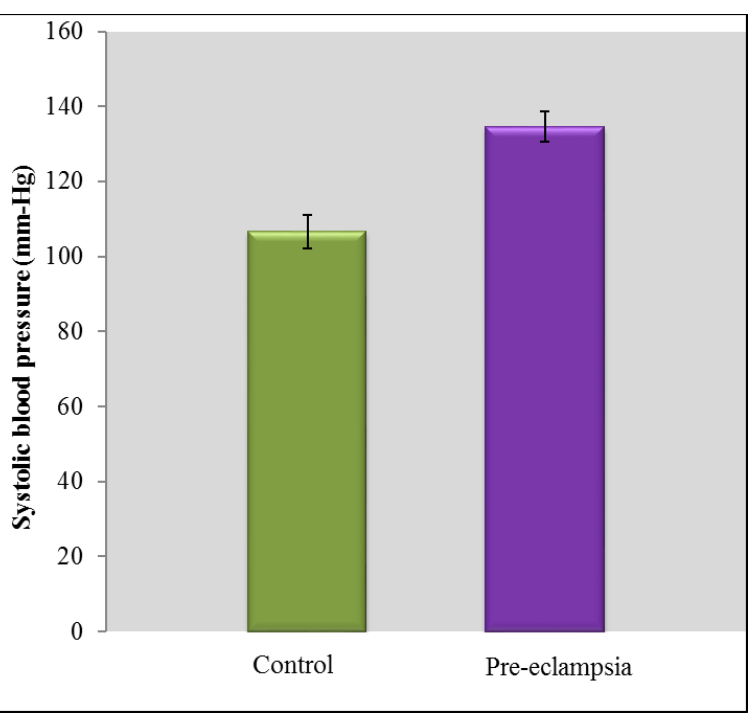

Fig. 1: SBP in cases and controls

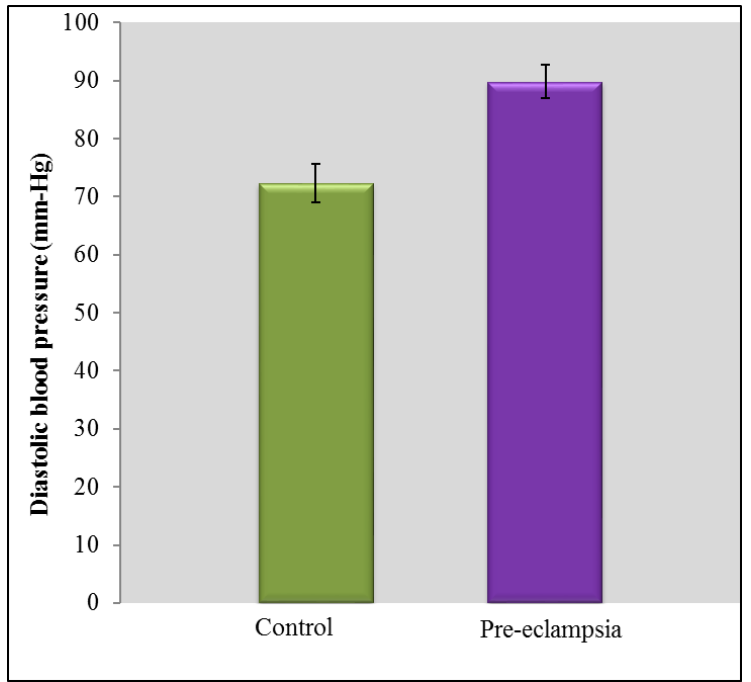

Fig. 2: DBP in cases and controls

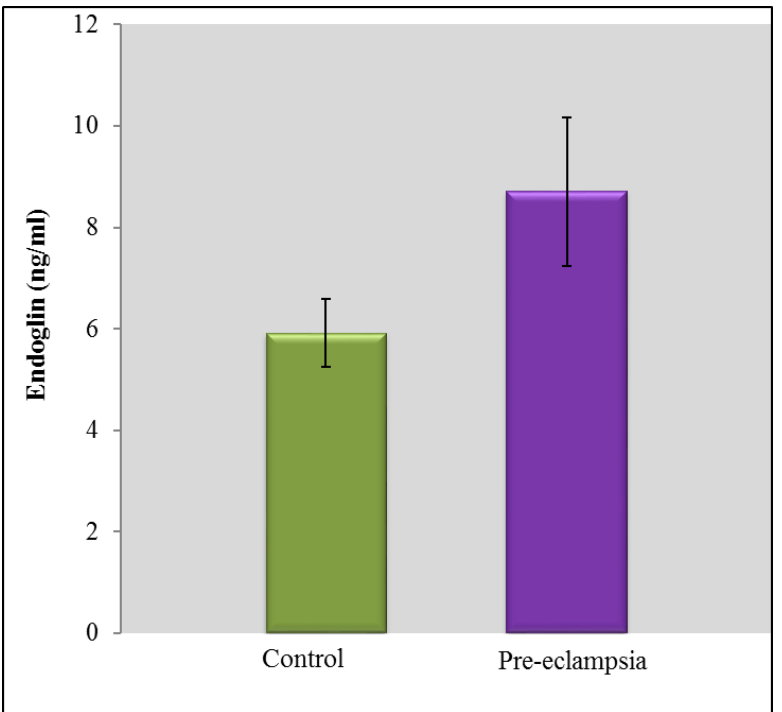

Fig. 3: sENG levels in cases and controls 


\section{Discussion}

Preeclampsia is defined as pregnancy specific disorder with SBP more than $140 \mathrm{~mm} \mathrm{Hg}$, DBP greater than $90 \mathrm{~mm} \mathrm{Hg}$ or proteinuria greater than $0.3 /$ day or + after twenty weeks of gestation. It is the major cause for maternal and foetal mortality and morbidity. Delivery is the only known cure for pre-eclampsia. The obstetricians have to support and take temporary measures in women who develop preeclampsia before term for a safer delivery of the baby. An early detection of the preeclampsia will decrease the adverse effect on foetal and maternal outcomes. If any method is identified, it would be of great clinical benefit.

In the present study, 40 patients had the mean systolic BP of $106.6 \pm 4.5$ and mean diastolic BP of $72.3 \pm 3.3$ and they are considered as control. The remaining 35 patients were observed with increased blood pressure. sENG levels were elevated in these patients. This shows a positive correlation between the concentration of sENG and blood pressure $(r$ value $=$ 0.746 for $\mathrm{SBP} \& \mathrm{r}$ value $=0.567$ for $\mathrm{DBP}$ ). The $\mathrm{p}$ value for systolic and diastolic blood pressure is 0.01 , which is significant. Out of 40 controls, 23 women had SBP level of $100-110 \mathrm{~mm} \mathrm{Hg}$ and 17 women with 110-120 $\mathrm{mm} \mathrm{Hg}$. The sENG level ranged between 5.1 and 7.1 $\mathrm{ng} / \mathrm{ml}$. Three controls had lower limit $5.1 \mathrm{ng} / \mathrm{ml}$ and their corresponding blood pressure was $100 / 70 \mathrm{~mm} \mathrm{Hg}$.

The systolic blood pressure ranged between 128 $140 \mathrm{mmHg}$ among pre eclamptic patients. The diastolic blood pressure ranged between $86-96 \mathrm{mmHg}$ among the cases. The mean DBP was $89.82 \pm 2.9$. The sENG level varied from $7.2-13.4 \mathrm{ng} / \mathrm{ml}$ with mean $8.7 \pm 1.4$. As a result, the blood pressure showed positive correlation with $\mathrm{sENG}$ ( $\mathrm{r}$ value $=0.729$ for SBP \& $\mathrm{r}$ value $=0.713$ for $\mathrm{DBP}$ ).

There are few studies which also have reported increased blood pressure in preeclampsia. Rana et al. had reported mean SBP $147 \pm 10$ and DBP $93 \pm 7$ in patients who developed preeclampsia in 2007. The mean sENG was $10.2 \pm 2.0$ in preeclampsia. There was a significant correlation with $\mathrm{sENG}$ and $\mathrm{BP}$ in their study. ${ }^{10}$ Sandrim et al. in 2008 had reported mean SBP $142.3 \pm 16.7$ and DBP $89.6 \pm 12.4$ in preeclamptic women with $\mathrm{p}$ value $<0.05 .{ }^{11}$ Recently, Elhawary et al. had reported with mean SBP $130.75 \pm 10.60$. The $p$ value is 0.038 , which is significant. The mean DBP is around $77 \pm 5.2$. The corresponding $\mathrm{p}$ value is 0.043 , which is significant. ${ }^{12}$ The mean DBP $(89.82 \pm 2.9)$ of present study is comparable with studies reported by Sandrim et al. ${ }^{11}$

There is a development of hypertension, leakage of proteins into the tissues and urine as a result of severe vasoconstriction. This is due to excess secretion of soluble ENG. Circulatory factors in combination with locally released mediators generate hypertension in the woman. There is also evidence from some animal preparations that indicate that placental ischaemia leads to the production of vasoactive substances that both cause vasoconstriction and inhibition of vasodilation leading to hypertension. The present study is also consistent with the above studies and proving that soluble endoglin as a role in predicting preeclampsia. Therefore, this study showed a positive correlation between soluble endoglin and blood pressure which was significant.

\section{Conclusions}

The present study showed that women with increased serum soluble endoglin levels developed preeclampsia which suggests the role of serum soluble endoglin. Serum soluble endoglin played a major role in predicting the onset of preeclampsia as it starts circulating few weeks prior to the symptoms. Correlation was achieved between systolic, diastolic blood pressure and serum endoglin levels. Hence, it is suggested that soluble endoglin will be useful in predicting preeclampsia. The main cause is endothelial dysfunction. Estimating a sensitive and specific serum biomarker soluble endoglin for preeclampsia along with urine protein analysis will not only improve the accuracy, but also expedite the diagnosis of preeclampsia.

\section{References}

1. Ashley K. Woods, Darren S. Hoffmann, Christine J. Weydert, Scott D. Butler, Yi Zhou, Ram V. Sharma, Robin L. Davisson. Adenoviral delivery of VEGF $_{121}$ early in pregnancy prevents spontaneous development of preeclampsia in BPH/5 mice. Hypertension. 2011;57;94102.

2. Mark A. Brown, Marshall D. Lindheimer, Michael de Swiet, Andre Van Assche, and Jean-Marie Moutquin The classification and diagnosis of the hypertensive disorders of pregnancy: statement from the international society for the study of hypertension in pregnancy (ISSHP). Hypertension in pregnancy. 20(I). ix-xiv (2001)

3. Sarosh Rana, S. Ananth Karumanchi, Richard J. Levine, Shivalingappa Venkatesha, Jose Alejandro Rauh-Hain, Hector Tamez, Ravi Thadhani. Sequential Changes in Antiangiogenic Factors in Early Pregnancy and Risk of Developing Preeclampsia Hypertension. 2007;50:137142.

4. Magnussen EB, Vatten LJ, Lund-Nilsen TI, Salvesen KA, Davey Smith G and Romundstad PR. Prepregnancy cardiovascular risk factors as predictors of pre-eclampsia: population based cohort study. Br Med J. 2007;335(7627): 978

5. Leeman L, Fontaine P. Hypertensive disorders of pregnancy. American Family Physician. 2008;78(1):93100.

6. Maynard SE, Min JY, Merchan J, Lim KH, Li J, Mondal S, Libermann TA, Morgan JP, Sellke FW, Stillman IE, Epstein FH, Sukhatme VP and Karumanchi SA. Excess placental soluble fms-like tyrosine kinase 1 (sFlt1) may contribute to endothelial dysfunction, hypertension, and proteinuria in preeclampsia. J Clin Invest. 2003;111(5):649-658. 
7. Anne Cathrine Staff, MD, PhD; Kristin Braekke, MD; Guro M. Johnsen, MSc; S. Ananth Karumanchi, MD; Nina Kittelsen Harsem, MD. Circulating concentrations of soluble endoglin (CD105) in fetal and maternal serum and in amniotic fluid in preeclampsia. Am J Obstet Gynecol. 2007;176-178.

8. Venkatesha S, Toporsian M, Lam C. Soluble endoglin contributes to the pathogenesis of preeclampsia. Nat Med. 2006;12(6):642-649.

9. Levine RJ, Lam C, Qian C, Yu KF, Maynard SE, Sachs BP, Sibai BM, Epstein FH, Romero R, Thadhani R and Karumanchi SA. Soluble endoglin and other circulating antiangiogenic factors in preeclampsia. $N \mathrm{Engl} \mathrm{J} \mathrm{Med}$. 2006;355(10):992-1005.

10. Rana S, Karumanchi SA, Levine RJ, Venkatesha S, Rauh-Hain JA, Tamez H, Thadhani R. Sequential changes in antiangiogenic factors in early pregnancy and risk of developing preeclampsia. Hypertension. 2007;50:137-142.
11. Sandrim VC, Palei ACT, Metzger IF, Gomes VA, Cavalli RC, Tanus-Santos JE. Nitric oxide formation is inversely related to serum levels of antiangiogenic factors soluble fms-like tyrosine kinase- 1 and soluble endoglin in preeclampsia. Hypertension. 2008;5 2:402-407.

12. Elhawary TM, EI-Bendary AS, Demerdash H. Materal serum endoglin as an early marker of pre-eclampsia in high risk patients. Int J Women's Health. 2012;4:521-525.

How to cite this article: Archana A. Brindha G, Sampson U. Studies on serum soluble endoglin: An indicator for preeclampsia. Int $\mathbf{J}$ Clin Biochem Res. 2018;5(4):599-603. 